\title{
On the Non-Euclidean Analogues of Tarry's Point.
}

\author{
By J. P. Gabbatr.
}

(Received 5th April 1923. Read 9th June 1923.)

\$1. Introductory. 1.1. The point known in elementary geometry by the name of Tarry* was first discussed by that writer as the point of concurrence of the perpendiculars respectively from the vertices of the base-triangle to the corresponding sides of Brocard $\mathrm{s}$ first triangle. Tarry's point is the point of the circumcircle diametrically opposite to Steiner's point, which is the fourth common point of the circumcircle and Steiner's circumellipse *

Tarry's point possesses the further property (due to Neuberg) that the three perpendiculars from that point to the sides of the basetriangle meet the three sides of that triangle at nine points which lie three by three in three further lines. Steiner's circumellipse is $\dagger$ the locus of a point which has the similar property that the lines through that point parallel to the sides of the base-triangle meet those sides at six points which lie three by three in two lines; the tangents to the ellipse at the vertices of the base-triangle are therefore respectively parallel to the opposite sides.

1.2. Berkhan states $\ddagger$ (without proof) that in non-euclidean geometry there are three points analogous to Tarry's point. It will be proved below that this theorem holds as regards Neuberg's property. For convenience the non-euclidean terminology will be used in the main; but it will be shewn that, as is frequently the case, $\|$ this expedient obscures the fact that the properties concerned belong to the general plane cubic, and usually occur in groups of three (cf. 3.4, below).

It is known $\left(v .{ }^{*} \S 17 \|\right)$ that in non-euclidean geometry the locus of a point $(M)$ such that the feet $(I, J, K)$ of the perpendiculars from $M$ to the sides $\left(B_{1} C_{1}, C_{1} A_{1}, A_{1} B_{1}\right)$ of a given plane triangle are in line is an order-cubic $\left(T_{0}\right)$ containing the

- Neuberg, Mathesis (1) 6 (1886), pp. 5-7.

† Neuberg, ibid. (1) 1 (1881), p. 184; (1) 2 (1882), pp. 42-6.

$\ddagger$ Berkhan, Arch. d. Math. u. Phys. (3) 11 (1907), p. 18.

|I V. Gabbatt, Proc. Camb. Phil. Soc. 21 (1922-3), pp. 297-362. References distinguished by an asterisk [thus : $\left.\left({ }^{*} 4.1\right)\right]$ are to that paper. 
points $A_{1}, B_{1}, C_{1}$ and the absolute poles $\left(A_{2}, B_{2}, C_{2}\right)$ of the lines $B_{1} C_{1}, C_{1} A_{1}, A_{1} B_{1}$. In $\$ 2$ of the present paper are discussed some of the properties of two other order-cubics each containing the six points $A_{1}, B_{1}, C_{1}, A_{2}, B_{2}, C_{2}$. One of these cubics $(U)$ is tha locus of a point $M$ such that the meets of the lines $M I, M J, M K$ with $C_{1} A_{1}, A_{1} B_{1}, B_{1} C_{1}$ respectively are in line ; and the other ( $\left.V\right)$ is the locus of a point $M$ such that the meets of $M I, M J, M K$ with $A_{1} B_{1}, B_{1} C_{1}, C_{1} A_{1}$ respectively are in line. The mutual relations of the cubics $U, V$ (the by-pedal loci for the triangle $A_{1} B_{1} C_{1}$ ) closely resemble the properties of the cubic $T_{0}(C f .2 .1,2.2)$.

In $\$ 3$ the analogues of Tarry's point are introduced as the common points (other than $A_{1}, B_{1}, C_{1}, A_{2}, B_{2}, C_{2}$ ) of the three cubics $T_{0}, U, V$. The place of an analogue of Steiner's circumellipse in the theory is also indicated.

\$2. The By-Pedal Loci. 2.1. In non-euclidean geometry, if $A_{1}, B_{1}, C_{1}$ denote the vertices of any triangle and $A_{2}, B_{2}, C_{2}$ the absolute poles of the lines $B_{1} C_{1}, C_{1} A_{1}, A_{1} B_{1}$ respectively; then (* $\$ 17$ ) the locus of a point $M_{0}$ such that the lines $A_{2} M_{0}, B_{2} M_{0}$, $C_{2} M_{0}$ meet $B_{1} C_{1}, C_{1} A_{1}, A_{1} B_{1}$ respectively at three points in a line $(m)$ is the order-cubic $T_{0}$ on which $A_{1}, A_{2} ; B_{1}, B_{2} ; C_{1}, C_{2}$ are pairs of correspondents of the same species. The cubic $T_{0}^{\prime}$ is also the locus of a point $M_{0}$ such that the lines $A_{1} M_{0}, B_{1} M_{0}$, $C_{1} M_{0}$ meet $B_{2} C_{2}, C_{2} A_{2}, A_{2} B_{2}$ respectively at three points in a line $\left(m^{\prime}\right)$. If $m, m^{\prime}$ be termed respectively the $(1,2)$-and $(2,1)$ pedal lines of $M_{0}$, and if $M_{0}, M_{3}$ denote correspondents (of the given species) on $T_{0}$; then $m, m^{\prime}$ are also respectively the $(2,1)$ and (1, 2)-pedal lines of $M_{3}$.

The absolute poles of $m, m^{\prime}$ are a pair of correspondents $\left(M_{1}, M_{2}\right)$ of the given species on $T_{0}$.

The envelope of the $(1,2)$-or of the (2,1)-pedal lines of the points $M_{0}$ of $T_{0}$ is a class cubic, the absolute reciprocal of $T_{0}$. (2.11)

2.2. Now let $P$ denote any point such that the lines $A_{2} P, B_{2} P$, $C_{2} P$ meet $C_{1} A_{1}, A_{1} B_{1}, B_{1} C_{1}$ respectively at three points $(Y, Z, X$ respectively) in a line $(n)$. Then by the quadrilateral property $P\left(A_{1}, X ; B_{1}, Y ; C_{1}, Z\right)$ or what is the same thing $P\left(A_{1}, C_{2}\right.$; $\left.B_{1}, A_{2} ; C_{1}, B_{2}\right)$ specifies conjugate pairs of rays in an involution pencil ; thus ${ }^{*} P$ is a point of the order-cubic $(U)$ on which $A_{1}, C_{2}$; $B_{1}, A_{2} ; C_{1}, B_{2}$ are pairs of correspondents of the same species. 
Again, if $P, P^{\prime}$ denote correspondents (of the given species) on $U$, then $A_{1}\left(B_{1}, A_{2} ; C_{1}, B_{2} ; P, P^{\prime}\right)$ specifies conjugate pairs of rays in an involution pencil; whence, if $Z^{\prime}$ denote the meet of the lines $n, A_{1} P^{\prime}$, then

$$
\begin{gathered}
P\left(A_{1} A_{2} B_{2} Z^{\prime}\right) \equiv P\left(A_{1} Y Z Z\right) \pi A_{1}\left(P Y Z Z^{\prime}\right) \\
\equiv A_{1}\left(P C_{1} B_{1} P^{\prime}\right) \pi A_{1}\left(P^{\prime} B_{2} A_{2} P\right) \equiv A_{1}\left(Z^{\prime} B_{2} A_{2} P\right),
\end{gathered}
$$

and thus $Z^{\prime}$ is on $A_{2} B_{2}$; similarly the meets of $n$ with the lines $B_{1} P^{\prime}, C_{1} P^{\prime}$ are on $B_{2} C_{2}, C_{2} A_{2}$ respectively; i.e., the lines $A_{1} P^{\prime}, B_{1} P^{\prime}, C_{1} P^{\prime}$ meet $A_{2} B_{2}, B_{2} C_{2}, C_{2} A_{2}$ respectively at three points on the line $n$. Thus: If $A_{1}, B_{1}, C_{1}$ denote the vertices of any triangle, and $A_{2}, B_{2}, C_{2}$ the absolute poles of the lines $B_{1} C_{1}$, $C_{1} A_{1}, A_{1} B_{1}$ respectively; then the locus of a point $P$ such that the lines $A_{2} P, B_{2} P, C_{2} P$ meet $C_{1} A_{1}, A_{1} B_{1}, B_{1} C_{1}$ respectively at three points in a line $(n)$ is the order-cubic $(U)$ on which $A_{1}, C_{2} ; B_{1}, A_{2}$; $C_{1}, B_{2}$ are pairs of correspondents of the same species. $\dagger$ The cubic $U$ is also the locus of a point $P$ such that the lines $A_{1} P, B_{1} P, C_{1} P$ meet $A_{2} B_{2}, B_{2} C_{2}, C_{2} A_{2}$ respectively at three points in a line $\left(n^{\prime}\right)$ If $n_{1} n^{\prime}$ be termed respectively the $(+1,2)$-and $(-2,1)$-by-pedal lines of $P$, and if $P, P^{\prime}$ denote correspondents (of the given species) on $U$; then $n, n^{\prime}$ are respectively the $(-2,1)$ and $(+1,2)$-by-pedal lines of $\boldsymbol{H}^{\prime \prime}$.

Now if the lines $A_{2} P, B_{2} P, C_{2} P$ meet $C_{1} A_{1}, A_{1} B_{1}, B_{1} C_{1}$ respectively at three points on $n$, and if $N, p$ respectively denote the absolute pole and polar of $n, P$; then, by reciprocation $q .+$ the Absolute, the lines $A_{2} N, B_{2} N, C_{2} N$ meet $A_{1} B_{1}, B_{1} C_{1}, C_{1} A_{1}$ respectively at three points on $\%$. Hence, as in (2.21), $N$ is a point of the order-cubic $(V)$ on which $A_{1}, B_{2} ; B_{1}, C_{2} ; C_{1}, A_{2}$ are pairs of correspondents of the same species. Again, if $N^{\prime}$ denote the absolute pole of $n^{\prime}$; then similarly the lines $A_{1} N^{\prime}, B_{1} N^{\prime}, C_{1} N^{\prime}$ meet $C_{2} A_{2}, A_{2} B_{2}, B_{2} C_{2}$ at three points on $p$. The points $N, N^{\prime}$ are therefore correspondents (of the given species) on $V$, and there is complete reciprocity between the properties of the cubics $U, V$. Thus: The locus of a point $N$ such that the lines $A_{2} N, B_{2} N, C_{2} N$ meet $A_{1} B_{1}, B_{1} C_{1}, C_{1} A_{1}$ respectively at three points in a line $(p)$ is the order-cubic (V) on which $A_{1}, B_{8} ; B_{1}, C_{2} ; C_{1}, A_{2}$ are pairs of corre-

* Cayley, Liouville, 9 (1844), p. $285=$ Papers, 1, r. 183.

+ A case of a theorem due to Grassmann and Clebsch ; v. (* 17.1).

¥ The symbol q. signifies with respect to. 
spondents of the sams species. ${ }^{*}$ The cubic $\nabla$ is also the locus of a point $N$ such that the lines $A_{1} N, B_{1} N, C_{1} N$ meet $C_{2} A_{2}, A_{3} B_{2}$, $B_{2} C_{2}$ respectively at three points in a line $\left(p^{\prime}\right)$. If $p, p^{\prime}$ be termed respectively the $(-1,2)$-and $(+2,1)$-by-pedal lines of $N$, and if $N, N^{\prime}$ denote correspondents (of the given species) on $V$; then $p, p^{\prime}$ are respectively the $(+2,1)$-and $(-1,2)$-by-pedal lines of $N^{\prime}$.

If $P, P^{\prime}$ denote any pair of correspondents (of the given species) on $U ; p, p^{\prime}$ respectively the absolute polars of $P, P^{\prime} ; n, n^{\prime}$ respectively the $(+1,2)$-and $(-2,1)$-by-pedal lines of $P$; and $N, N^{\prime}$ respectively the absolute poles of $n, n^{\prime}$; then $N, N^{\prime}$ are correspondents (of the given species) on $V$, and $p, p^{\prime}$ are respectively the $(-1,2)$-and $(+2,1)$-by-pedal lines of $N$

The envelope of the $(+1,2)$-or of the $(-2,1)$-by-pedal lines of the points on $U$ is a class-cubic, the absolute reciprocal of $V$; and reciprocally for $V, U$.

2.3. The writer has shewn $\left({ }^{*} 17.11\right)$ that, if $M_{0}$ denote any point on the cubic $T_{0}(2.11)$, then the perpendiculars at $A_{1}, B_{1}, C_{1}$ to the lines $A_{1} M_{0}, B_{1} M_{0}, C_{1} M_{0}$ respectively meet at a point $M_{2}$; the perpendiculars at $A_{2}, B_{2}, C_{2}$ to the lines $A_{2} M_{0}, B_{2} M_{0}, C_{2} M_{0}$ meet at a point $M_{1}$; and $M_{1}, M_{2}$ are correspondents (of the same species as $A_{1}, A_{2}$ ) on $T_{0}$. These properties are analogous to the rectangular property of the euclidean circle. It may be shewn that the cubics $U, V$ possess corresponding mutual relations.

For if $P$ denote any point of $U$, then (2.22) the lines $A_{1} P, A_{2} B_{2}$ meet (at $Z^{\prime \prime}$, say) on the absolute polar of a point $\left(N^{\prime}\right)$ on $V . Z^{\prime \prime}$ is thus the absolute pole of the line $C_{1} N^{\prime}$, which is therefore perpendicular to $A_{1} P$; similarly the lines $A_{1} N^{\prime}, B_{1} N^{\prime}$ are perpendicular to $B_{1} P, C_{1} P$ respectively. Thus: If $P$ denote any point on the cubic $U$, then the lines through $C_{1}, A_{1}, B_{1}$ perpendicular to $A_{1} P$, $B_{1} P, C_{1} P$ respectively meet at a point $N^{\prime}$; the lines through $B_{2}, C_{2}$, $A_{2}$ perpendicular to $A_{2} P, B_{2} P, C_{2} P$ respectively meet at a point $N$; and $N, N^{\prime}$ are correspondents (of the given species) ori $V$. Similarly for $V, U$.

Again, it is well known that the lines $A_{1} A_{2}, B_{1} B_{2}, C_{1} C_{2}$ meet at a point $(H)$, the common orthocentre of the triangles $A_{1} B_{1} C_{1}$, $A_{2} B_{3} C_{2}$; and that the lines $B_{1} C_{1}, C_{1} A_{1}, A_{1} B_{1}$ meet $B_{2} C_{2}, C_{2} A_{2}$, $A_{2} B_{2}$ respectively at three points $\left(A_{0}, B_{0}, C_{0}\right.$ respectively) in a line,

* A case of a theorem due to Grassmann and Clebsch; v. (* 17.1). 
the common orthaxis of the two triangles. The writer has shewn $\left({ }^{*} 8.4,{ }^{*} 15\right)$ that the Absolute is the pole conic of the orthaxis q. a class-cubic $\left(\Theta_{3}\right)$ of which $T_{0}$ is the Cayleyan; $\theta_{3}$ being the classcubic q. which the point-pairs $A_{1}, A_{2} ; B_{1}, B_{2} ; C_{1}, C_{2}$ are degenerate pole conics. It is easy to prove somewhat similar theorems for the cubies $U, V$.

For there is an order-cubic $\left(U^{\prime}\right)$ q. which $A_{1}, C_{2} ; B_{1}, A_{2} ; C_{1}, B_{2}$ are pairs of conjugate poles; $U$ being the Hessian of $U^{\prime}$. Let the polar conic, q. $U$, of any point $M$ be denoted by $S(M)$. Then $S\left(A_{2}\right)$ consists of a pair of lines meeting at $B_{1}$; and since $B_{2}, C_{1}$ are conjugate poles q. $U^{\prime}$, therefore the pair of lines $B_{1} B_{2}, B_{1} C_{1}$ are harmonic q. the pair of lines $S\left(A_{2}\right)$. Thus $B_{1} C_{1}$ is the polar of $H$ q. $S\left(A_{2}\right)$, and is therefore the polar of $A_{2}$ q. $S(H)$; similarly the lines $C_{1} A_{1}, A_{1} B_{1}$ are the polars, q. $S(H)$, of $B_{2}, C_{2}$ respectively, and thus: The Absolute is the polar conic of the orthocentre of the triangle $A_{1} B_{1} C_{1}$ q. either of two order cubics $\left(U^{\prime}, V^{\prime}\right)$ of which $V, V$ are respectively the Hessians.

Now any pair of correspondents (of the given species) $N, N^{\prime}$ on $V$ are conjugate points q. every polar conic q. $V^{\prime}$, and in particular q. the Absolute; whence (2.22): The $(+1,2)$-and $(-2,1)-b y$ pedal lines of any point $P$ on $U$ are perpendicular; and similarly for $V$.

\$3. The Analogues of Tarry's Point. 3.1. The six points $A_{1}$, $B_{1}, C_{1}, A_{2}, B_{2}, C_{2}$ are common to the cubics $T_{0}, U, V$. If $M$ denote any other common point of $T_{0}$ and $U$, and $I, J, K$ the feet of the perpendiculars from $M$ to $B_{1} C_{1}, C_{1} A_{1}, A_{1} B_{1}$ respectively; then (2.11) three of the nine points common to the three lines $M I, M J, M K$ and the three lines $B_{1} C_{1}, C_{1} A_{1}, A_{1} B_{1}$ are in a seventh line, and (2.21) three more of the nine points are in an eighth line. The remaining three of the nine points are therefore in a ninth line, and thus (2.22) $M$ is a point of the cubic $V$. Hence: The cubics $T_{0}, U, V$ have three points $\left(P_{3}, Q_{3}, R_{3}\right)$ other than $A_{1}$, $B_{1}, C_{1}, A_{2}, B_{3}, C_{2}$ common to all three. The points $P_{3}, Q_{3}, R_{3}$ and no other points are such that the three perpendiculars from any one of them to the sides of the triangle $A_{1} B_{1} C_{1}$ are definite and meet the three sides at nine distinct points which lie three by three in three further lines. $P_{3}, Q_{3}, R_{3}$ are similarly related to the triangle $A_{2} B_{2} C_{2}$. 
It follows from (2.31) that: If and only if $M$ denote one of the three points $P_{3}, Q_{3}, R_{3}$; then the three perpendiculars to the lines $A_{1} M, B_{1} M, C_{1} M$ from $A_{1}, B_{1}, C_{1}$ respectively, from $B_{1}, C_{1}, A_{1}$ respectively, and from $C_{1}, A_{1}, B_{1}$ respectively are concurrent.* The three points of concurrence are on $T_{0}, U, V$ respectively. Similarly for $M ; A_{2}, B_{2}, C_{2}$; and $T_{0}, V, U$.

3.2. The points $A_{1}, B_{1}, C_{1}$ are respectively the correspondents of one species (on $T_{0}$ ); and the points $A_{2}, B_{2}, C_{2}$ respectively the correspondents of another species (on $T_{0}$ ) of the points $A_{0}, B_{0}, C_{0}$ (2.32). If the lines $B_{1} C_{2}, C_{1} A_{2}, A_{1} B_{2}$ meet $B_{2} C_{1}, C_{2} A_{1}, A_{2} B_{1}$ respectively at $A_{3}, B_{3}, C_{3}$; then $\left({ }^{*} 5.1\right)$ the points $A_{3}, B_{3}, C_{3}$ are the correspondents of the third species (on $T_{0}$ ) of $A_{0}, B_{0}, C_{0}$ respectively, and the lines $B_{3} C_{3}, C_{3} A_{3}, A_{3} B_{3}$ contain $A_{0}, B_{0}, C_{0}$ respectively. More generally, if $A_{l}, B_{l}, C_{l}$ denote respectively the correspondents (of a given species) of $A_{0}, B_{0}, C_{0}$; then $A_{m}, B_{m}, C_{m}$ are respectively the correspondents (of that species) of $A_{n,} B_{n}, C_{n}$ $(l, m, n=1,2,3)$.

Further, if $O, P, Q, R, S$ denote any five points on an ordercubic, and $P^{\prime}, Q^{\prime}, R^{\prime}, S^{\prime \prime}$ respectively the correspondents of any (the same) species of $P, Q, R, S$; then $\dagger$

$$
O(P Q R S) \pi O\left(P^{\prime} Q^{\prime} R^{\prime} S^{\prime}\right)
$$

Thus, if $M$ denote any one of the three points $P_{3}, Q_{3}, R_{3}(3.11)$, then (3.21) $A_{3}\left(A_{0} B_{3} C_{3} M\right) \pi M\left(A_{0} B_{3} C_{3} A_{3}\right)$

$$
\begin{aligned}
& \pi M\left(A_{1} B_{2} C_{2} A_{2}\right) \\
& \pi M\left(B_{2} A_{1} B_{1} C_{1}\right) \\
& \pi M\left(B_{3} A_{0} B_{0} C_{0}\right) \\
& \pi B_{3}\left(M A_{0} B_{0} C_{0}\right) \\
& \equiv B_{3}\left(M C_{3} B_{0} A_{3}\right),
\end{aligned}
$$

whence the conic which contains $M, B_{3}, C_{8}$ and touches the line $A_{0} A_{3}$ at $A_{3}$ also touches the line $B_{0} B_{3}$ at $B_{3}$; similarly the conic touches the line $C_{0} C_{3}$ at $C_{3}$. Thus: There is a conic which touches the lines $A_{0} A_{3}, B_{0} B_{3}, C_{0} C_{3}$ at $A_{3}, B_{3}, C_{3}$ respectively, and contains the points $P_{3}, Q_{3}, R_{3}$ specified in (3.11).

It follows that $P_{3}, Q_{3}, R_{3}$ are respectively correspondents (of

- The euolidesn analogue is due to Nenberg, Mathesio (1) 5 (1885), p. 208.

† Cayley, loc. cit. (2.2). 


\section{4}

the same species as the pair of correspondents $A_{1}, A_{2}$ ) of three points $P_{0}, Q_{0}, R_{0}$ in line on the cubic $T_{0}$. If $T_{3}$ be the order-cubic apolar $\left({ }^{*} 1.3\right)$ to $\theta_{3}(2.32)$; then the conic specified in (3.23) is the mixed poloconic ( $\left.{ }^{*} 10.1\right)$ q. $T_{3}$ of the orthaxis $A_{0} B_{0} C_{0}$ and another line; it is also the mixed poloconic $\left({ }^{*} 10.6\right)$ q. $\theta_{3}$ of the orthocentre $H$ and another point.

3.3. Now let $Q$ denote any point such that the lines $A_{0} Q, B_{0} Q$, $C_{0} Q$ meet the lines $C_{3} A_{3}, A_{3} B_{3}, B_{3} C_{3}$ at three points $(E, F, D$ respectively) in line ; then $A_{0} Q, B_{0} Q, C_{0} Q$ meet $A_{3} B_{3}, B_{3} C_{3}, C_{3} A_{3}$ respectively at three other points in line. Further. by the quadrilateral property $Q\left(A_{3}, A_{0} ; B_{3}, B_{0} ; C_{3}, C_{0}\right)$, and $Q\left(A_{3}, D ; B_{3}, E\right.$; $\left.C_{3}, F^{\prime}\right)$ or what is the same thing $Q\left(A_{3}, C_{0} ; B_{3}, A_{0} ; C_{3}, B_{3}\right)$, specify conjugate pairs of rays in involution pencils. Thus $Q\left(A_{0} B_{3} C_{3} A_{3}\right)$ $\pi Q\left(B_{3} A_{0} B_{0} C_{0}\right) \pi Q\left(B_{0} A_{3} B_{3} C_{3}\right)$, whence as in (3.23): The locus of a point $Q$ such that the lines $A_{0} Q, B_{0} Q, C_{0} Q$ meet the lines $B_{3} C_{3}$, $C_{3} A_{3}, A_{3} B_{3}$ at six points (other than $A_{0}, B_{0}, C_{0}$ ) which lie three by three in two lines is the conic specified in (323).

3.4. Discarding the non-euclidean terminology, we may generalise the theorems $(3.11,3.23,3.31)$ as follows: Let $A_{0}, B_{0}, C_{0}$ denote any three collinear points on any order-cubic $T_{0}$, and $A_{n}, B_{n}$, $C_{n}$ correspondents (of the same species) of $A_{0}, B_{0}, C_{0}$ respectively $(n=1,2,3)$. Then there are three and only three points $\left[G_{l}\right]$ such that the lines $A_{m} G_{l}, B_{m} G_{l}, C_{m} G_{l}$ are definite and meet the lines $B_{n} C_{n}, C_{n} A_{n}, A_{n} B_{n}$ at nine distinct points which lie three by three in three further lines. The lines $A_{n} G_{l}, B_{n} G_{l}, C_{n} G_{l}$ also meet the lines $B_{m} C_{m}, C_{m} A_{m}, A_{m} B_{m}$ at nine distinct points which lie three by three in three further lines. The three points $\left[G_{l}\right]$ are contained by the cubic $T_{0}$ and also by the conic $\left(s_{l}\right)$ which touches the lines $A_{0} A_{l}, B_{0} B_{l}, C_{0} C_{l}$, at $A_{l}, B_{l}, C_{l}$ respectively. The correspondents (associated with the suffix $l$ ) on $T_{0}$ of the three points $\left[G_{l}\right]$ are in line. The conic $s_{l}$ is the locus of a point $Q$. such that the lines $A_{0} Q, B_{0} Q$, $C_{0} Q$ meet the lines $B_{\imath} C_{\imath}, C_{\imath} A_{\imath}, A_{\imath} B_{\imath}$ al six points (other than $A_{0}$, $\left.B_{0}, C_{0}\right)$ which lie three by three in two lines $(l, m, n=1,2,3)$.

It is to be observed that the lines of collinearity $\left(g_{1}, g_{2}, g_{3}\right)$ of the $(1,2,3)$-correspondents respectively of the three groups of three points $\left[G_{1}\right],\left[G_{2}\right],\left[G_{3}\right]$ are not in general identical. It may be proved that the tangents to $T_{0}$ at $A_{n}, B_{n}, C_{n}$ determine a triangle in perspective with the triangle $A_{n} B_{n} C_{n}$, and that $g_{n}$ is the polar, 


\section{5}

q. the triangle $A_{n} B_{n} C_{n}$, of the centre of perspective of the two triangles $(n=1,2,3)$.

3.5. In the euclidean case $\left(c f .{ }^{*} \$ 16\right)$, if $A_{1} B_{1} C_{1}$ remains an actual triangle (the base-triangle), then $T_{0}$ breaks up into the circumcircle of that triangle and the line at infinity, and each of the cubics $U, V$ breaks up into a circumconic of the base-triangle* and the line at infinity. $A_{0}, B_{0}, C_{0}$ become the points at infinity of the lines $B_{1} C_{1}, C_{1} A_{1}, A_{1} B_{1}$ respectively, and $A_{3}, B_{3}, C_{3}$ become the points of the circumcircle diametrically opposite to $A_{1}, B_{1}, C_{1}$ respectively. The conic specified in (3.23) becomes the Steiner circumellipse of the triangle $A_{3} B_{3} C_{3} ; \dagger$ and, of the points $P_{3}, Q_{3}$, $R_{3}$ specified in (3.11), one becomes the Tarry point of the basetriangle, and the other two become the points at infinity on the ellipse just specified.

* Neuberg, Mathesis (1) 3 (1883), p. 144 ; ibid. (1) 5 (1885), p. 208 ; ibid. (1) 6 (1886), p. 5. Cf. the remark Encyk d. math. Wiss. III. AB 10, p. 1266, Il. 2-7.

† The conic $s_{1}$ specitied in (3.41) becomes the Steiner circumellipse of the base-triangle. 